\title{
Paradoxical Air Embolism Post C.V Catheter Removal
}

\author{
Abhishek B* and Mohit M \\ Department of Anaesthesiology and Critical Care, Fortis Hospital, India \\ *Corresponding Author: Abhishek Biswas, Department of Anaesthesiology and \\ Critical Care, Fortis Hospital, Mohali, Punjab, India, Tel: 9988523216; E-mail: \\ dr.abhishekbiswas13@gmail.com
}

\section{Letter to Editor \\ Volume 2 Issue 2}

Received Date: October 24, 2017

Published Date: November 06, 2017
Keywords: $\quad$ Paradoxical; $\quad$ Transthoracic echocardiogram; Air embolism

Abbreviations: VAE: Venous Air Ambolism; CVC: Central Venous Catheter; TTE: Transthoracic Echocardiogram; DVT: Deep Vein Thrombosis

\section{Letter to the Editor}

Definitive diagnosis of venous air embolism (VAE) following removal of central venous catheter (CVC) is difficult in spontaneously breathing patients. We report a patient in whom visualization of air bubbles in the right ventricle on transthoracic echocardiogram (TTE) along with clinical manifestations diagnosed probable VAE. A 50 year old ASA-1 gentleman operated for perforation peritonitis was on Inj. Fragmin 2500 I.U for deep vein thrombosis (DVT) prophylaxis. He was planned to shift to the ward after CVC removal (supine position after deep inspiration). Within 2-3 minutes of its removal and application of airtight dressing, patient had cough followed by dyspnea (respiratory rate increased from $30 / \mathrm{min}$ to $45 / \mathrm{min}$ ). Simultaneously, BP decreased from $120 / 78 \mathrm{~mm} \mathrm{Hg}$ to $85 / 50 \mathrm{mmHg}, \mathrm{SpO}_{2}$ decreased from $100 \%$ to $64 \%$ and heart rate increased from $86 /$ minute to $114 /$ minute. Normal saline $(500 \mathrm{~mL})$ and Mephentermine in incremental doses were administered to maintain BP. In addition, he was immediately placed in the left lateral decubitus position and high flow $\mathrm{O}_{2}$ was supplemented. Urgent bedside echocardiogram revealed multiple airbubbles in the right chamber of the heart. Auscultation of the heart and lungs was unremarkable. The chest X-ray revealed a radiolucent column along the lateral aspect of the right neck where his CVC had been placed. Electrocardiogram and troponin levels were within normal limits. D-dimer was $4 \mathrm{nmol} / \mathrm{l}$. Within 45 minutes, the hemodynamic and respiratory parameters returned to normal and rest of the hospital stay was uneventful. Therapeutic dosage of Inj. Fragmin 5000 I.U was administered and ICU stay was prolonged for one more day for observation. Though TEE is commonly employed, TTE has not been extensively used for VAE detection and such reports are scanty. Diagnosing VAE in intensive care requires a high degree of suspicion. Tachycardia, hypotension, desaturation and dyspnea are not uncommon in ICU from other causes. Our initial suspicion was pulmonary thromboembolism despite patient being on prophylactic dosage of anticoagulants. In addition, we also considered VAE as a possible cause for this acute event in view of cough and an association of CVC removal. Radiolucent column in chest X-ray and air bubbles in the right ventricle on TTE and an equal sized right ventricle to left ventricle with new onset tricuspid regurgitation prompted us to suspect VAE. The clinical manifestations were attributed to the entrained air in the pulmonary circulation and resultant mechanical obstruction of the right ventricular outflow tract and the pulmonary artery. Further, larger volume of air would have caused air lock and an acute cardiovascular and pulmonary collapse. The other possibilities considered were anaphylaxis and an acute intrinsic cardiac event. Anaphylaxis to the drug requires temporal relationship but no pharmacological agent or blood was administered before this episode. Although ECG was normal (only 15\% cases show classical S1Q3T3), D-dimer was borderline (low specificity) [1] clinical features, high Wells score and ECHO findings along with acute onset of events points towards VAE. We didn't have the option of CT Pulmonary Angiography due to financial restrains and gradual improvement of the patient. Though, the mortality rates have been reported from $23 \%$ to $50 \%$, absence of patent foramen ovale, prompt recognition and management were primarily responsible for the better patient outcome, despite significant air entrainment [2]. Hyperbaric oxygen and 


\section{Anaesthesia \& Critical Care Medicine Journal}

cardiopulmonary resuscitation are other therapeutic options in an event of massive VAE or neurologic symptoms. To conclude, CVC removal even in supine position, places the patients at risk of VAE. TTE is invaluable in its early diagnosis, management and subsequent evaluation.

\section{References}

1. Reka G Szigeti (2014) D-Dimer. Medscape.

2. Feil M (2015) Preventing central line air embolism. Am J Nurs 115(6): 64-69. 